\title{
Shifted Excitation Raman Difference Spectroscopy applied to extraterrestrial particles returned from the asteroid Itokawa
}

\author{
U. Böttger ${ }^{\text {a, *, M. Maiwald }}{ }^{\text {b }}$, F. Hanke ${ }^{\text {a, c }}$, M. Braune ${ }^{\text {b }}$, S.G. Pavlov ${ }^{\text {a }}$, S. Schröder ${ }^{\text {a }}$, I. Weber ${ }^{\text {d }}$, \\ H. Busemann ${ }^{\text {e }}$, B. Sumpf ${ }^{\text {b }}$, G. Tränkle ${ }^{\text {b }}$, H.-W. Hübers ${ }^{\text {a, c }}$ \\ ${ }^{a}$ Institute of Optical Sensor Systems, German Aerospace Center (DLR), Rutherfordstr. 2, 12489 Berlin, Germany \\ ${ }^{\mathrm{b}}$ Ferdinand-Braun-Institut, Leibniz Institut für Höchstfrequenztechnik, Gustav-Kirchhoff-Strasse 4, 12489 Berlin, Germany \\ ${ }^{\mathrm{c}}$ Humboldt-Universität zu Berlin, Institut für Physik, Newtonstr. 15, 12489 Berlin, Germany \\ d Institut für Planetologie, Wilhelm-Klemm-Str. 10, WWU Münster, 48149 Münster, Germany \\ ${ }^{\mathrm{e}}$ ETH Zürich, Institut für Geochemie und Petrologie, Clausiusstrasse 25, 8092 Zürich, Switzerland
}

\section{A B S T R A C T}

Two extraterrestrial particles from the asteroid Itokawa are investigated applying Shifted Excitation Raman Difference Spectroscopy (SERDS). These particles were returned by the Hayabusa mission of the Japanese Space Agency JAXA. For SERDS a diode laser based microsystem light source at $488 \mathrm{~nm}$ is used for excitation. It has been found that fluorescence signals masking the Raman spectral features of interest can be substantially separated by applying SERDS. Therefore, SERDS improves the information obtained from the Raman spectra and enables a reliable analysis for investigations on extraterrestrial samples.

\section{Ocis codes}

(300.6450) Spectroscopy, Raman; (300.6360) Spectroscopy, laser; (180.1790) Confocal microscopy; (180.5655) Raman microscopy; (140.5960) Semiconductor lasers; (140.3490) Lasers, distributed feedback; (140.3515) Lasers, frequency doubled; (060.2420) Fibers, polarization-maintaining; (060.3735) Fiber Bragg gratings.

\section{Introduction}

One of the main goals of space exploration activities is to return samples from extraterrestrial bodies in the Solar System such as the Earth Moon, Mars, asteroids, comets, and other planets and their satellites. Sample return missions have been successfully completed or are currently planned by leading space agencies NASA (May and Mattingly, 2011; Witze, 2014), ESA (Rull et al., 2013), JAXA (Tachibana et al., 2014; Yoshikawa et al., 2015), RSA (Zelenyi et al., 2014) and CNSA (Zheng et al., 2008). The major reason for extraterrestrial sample return is the in-depth analysis that is possible due to the availability of state-ofthe-art instrumentation in the laboratory that is not yet available on spacecraft.

However, many analytical techniques require sample preparation (e.g., polishing or coating), which can alter the precious original material to some extent. In order to maximize scientific return, it is essential to first perform non-destructive, or at least minimally-invasive, analytical methods, before modifying or destroying these unique samples. Raman spectroscopy is considered as a non-destructive method to investigate the molecular and crystal structure of a sample. It is non-destructive only if the laser power density at the sample is kept below damage threshold and the target material is stable with respect to moderate heating. Raman spectroscopy is based on inelastic scattering of monochromatic light, usually provided by a laser. The frequency shifts of the inelastically scattered laser photons provide the Raman spectrum of the sample. It is unique for each sample and thus allows its identification. Combined with confocal microscopy, Raman spectroscopy can be applied to investigate inclusions in translucent samples (Hooijschuur et al., 2013) or for measurements on samples placed behind a protecting cover window, because only radiation coming from the region in the focus of the excitation spot is collected and contributes to the measured Raman spectrum. A frequent problem for the interpretation of Raman spectra is a broadband fluorescence signal masking the comparatively narrow Raman bands. Fluorescence is caused by excitation of fluorophores that can occur naturally in biological materials as well as in minerals. Its strength depends on the exciting laser wavelength.

There are several possibilities to reduce the impact of fluorescence in Raman spectroscopy (Wei et al., 2015). A common way to obtain Raman spectra without fluorescence background comprises time-gated measurements with pulsed lasers. In this approach most of the

\footnotetext{
* Corresponding author.

E-mail address: ute.boettger@dlr.de (U. Böttger).
} 
fluorescence signal is not recorded if the detector operates in a gated mode and closes shortly after the Raman signal is detected (Matousek et al., 1999; Hooijschuur et al., 2013). However, time-gated Raman spectroscopy shows on the time scale an overlap of Raman and fluorescence signal which could still disturb the measurements and complicate the analysis of unknown samples. Additionally, it comes with other drawbacks such as the required high laser pulse intensities that might change mineral structures and the necessity for many repeated measurements in order to obtain Raman signals with significant signal-to-noise ratio. Another approach is based on choosing an excitation wavelength which causes minimal fluorescence such as 1064 $\mathrm{nm}$ (Tuschel, 2016). However, the Raman intensity is proportional to $1 /$ $\lambda^{4}$. As a consequence, Raman measurements in the near infrared spectral range require high excitation power up to the Watt level for sufficient signal intensity. Moreover, both methods imply either expensive extensions or a new instrument and are therefore not always the desired or feasible solution. To minimize the impact of fluorescence at low costs a method is preferred that is compatible to continuous wave (cw) Raman spectroscopy and can easily be adapted to existing systems. A demonstrated approach is Shifted Excitation Raman Difference Spectroscopy (SERDS) (Mosier-Boss et al., 1995; Zhao et al., 2002; Shreve et al., 1992; McCain et al., 2008; Maiwald et al., 2009a, 2014). This technique relies on the fact that the fluorescence spectrum is essentially independent of the excitation wavelength, if the excitation wavelengths are very close to each other. In contrast, the positions of lines or bands in the Raman spectrum depend on the excitation wavelength. Therefore, a slight change of the excitation wavelength will cause a slight shift of the spectral position of the Raman lines whereas the spectral position of the fluorescence signal remains unchanged. To utilize this effect for SERDS, two Raman spectra are measured under identical conditions using two excitation wavelengths slightly separated by typically $10 \mathrm{~cm}^{-1}$. Both Raman spectra are subtracted and a derivativelike SERDS spectrum is generated. Here, the desired Raman signals are efficiently separated from the fluorescence signal, and all required analysis can be performed using these SERDS spectra. An integration and baseline correction will lead to a Raman spectrum in a conventional form. It is worth noting that SERDS separates also Raman signals from other disturbing signals, such as ambient light (Maiwald et al., 2015) and fixed-pattern noise of multichannel detectors. A reconstruction of the Raman spectrum from its derivative is strictly speaking not necessary.

Up to this work SERDS has not been applied directly to samples from the Solar System, such as extraterrestrial particles from JAXA's sample return mission Hayabusa or fragments of meteorites. The particles obtained by the Hayabusa mission are particularly valuable and should not be contaminated. For specific research projects like for noble gas analysis it was even necessary that the particles stayed in a protective (inert $\mathrm{N}_{2}$ gas) atmosphere and never were exposed to Earth atmosphere. In these cases, where the Raman measurements were performed through a cover glass under which the particles were stored on a glass slide (Böttger et al., 2014a,b), SERDS would be a reasonable approach for minimizing fluorescence in Raman measurements.

In this work we upgraded a Raman microscope (WITec Alpha 300) for the application of SERDS by coupling the emission of a tunable microsystem diode laser source at $488 \mathrm{~nm}$ into the single-mode optical fiber which is connected to the microscope and is used as the excitation fiber. The compact diode laser module is developed by the FerdinandBraun-Institute and is suitable for Raman spectroscopy (Maiwald et al., 2015) and SERDS (Maiwald et al., 2009b). Two extraterrestrial particles returned by JAXA's Hayabusa mission to the asteroid Itokawa (Böttger et al., 2014a,b) were investigated with SERDS at $488 \mathrm{~nm}$ at the above mentioned microscope. The SERDS spectra are compared with conventional Raman spectra of the same particles. It is shown how SERDS can help to mitigate the problem of fluorescence and, therefore, makes the information obtained from the Raman spectra more reliable.

\section{Samples: particles from the asteroid Itokawa}

Hayabusa is a sample return mission from JAXA that returned samples from the S-type asteroid 25,143 Itokawa in 2010. The first studies indicated that Itokawa consists mostly of material with the petrologic type LL5-6 (Nakamura et al., 2011). Within the scope of JAXA's first two "International Announcements of Opportunity", a consortium study was performed for the analysis of noble gases in Itokawa samples (Busemann et al., 2013) combined with Raman spectroscopy, infrared spectroscopy (IR), and synchrotron radiation X-ray tomographic microscopy (SRXTM) (Meier et al., 2014). The latter methods yield grain mineralogy, density, and structure necessary to estimate the gas concentrations of potentially present cosmogenic, solar, trapped and radiogenic noble gases.

JAXA provided several particles with different investigation history to the consortium: The typical size of the samples is $50-100 \mu \mathrm{m}$. Some particles, embedded in epoxy, were investigated before allocation by Scanning Electron Microscopy (SEM), Time-of-Flight Secondary Ion Mass Spectrometry (ToF-SIMS) (Naraoka et al., 2012) or examined by neutronactivation analysis (Ebihara et al., 2011). These particles were exposed to Earth atmosphere but remained under a cover glass to prevent loss, damage and further contamination. Furthermore, several particles were delivered in inert $\mathrm{N}_{2}$ gas with no previous contact to Earth atmosphere (Busemann et al., 2015). For Raman spectroscopy measurements the transport container was equipped with a laser-transparent window (Böttger et al., 2014a,b).

Some particles showed remnants of carbon and gold due to a previous preparation procedure. These residues caused strong fluorescence that masked the Raman spectra making any interpretation very challenging. Moreover, further difficulties were encountered for the particles with carbon remnants, because significant laser-induced heating of these small particles due to absorption of the remaining carbon during Raman measurements could affect the subsequent noble gas analysis (Busemann et al., 2013, 2015; Meier et al., 2014).

Here, measurements on two Hayabusa samples are presented: Hayabusa RA-QD-02-0039 (\#39) and RA-QD-02-0068 (\#68) (Fig. 1). The particles were embedded in resin and a carbon fiber was attached, clearly visible in the photograph of particle \#39 in Fig. 1 left, which was required for X-ray microscopy. The brownish colored squares around the particles in Fig. 1 were the result of the different irradiation methods (SEM, SIMS), sputtering, coating, polishing and the potential deposition of additional material upon irradiation.

The methods applied to the particles prior to the Raman measurements are summarized in Table 1 . To avoid the use of water for polishing, alumina powder suspended in a mixture of ethanol, ethylene glycol, and glycerol was used (Nakamura et al., 2011). Each of these treatments may increase the probability of fluorescence during Raman spectroscopy.

\section{Experimental setup}

A compact semiconductor laser-based microsystem $488 \mathrm{~nm}$ light source, which provides two slightly different emission lines, was used for Raman spectroscopy and SERDS. It is based on second harmonic generation (SHG) of near-infrared laser emission. A distributed feedback ridgewaveguide diode laser with an emission at $976 \mathrm{~nm}$ was applied as a wavelength-stabilized pump light source. Cylindrical micro-optics are implemented for beam shaping and coupling of the pump light into a waveguide crystal for efficient SHG. Two additional micro-optical elements collimated the frequency-doubled beam at the exit. A more detailed description of the diode laser source is presented in (Maiwald et al., 2009a,b). The combined temperature management for the pump laser and SHG-crystal of the SHG microsystem $488 \mathrm{~nm}$ light source as used in this work opens the possibility of spectral tuning by changing the heatsink temperature of the device. The frequency converted excitation wavelength is changed with about $0.04 \mathrm{~nm} / \mathrm{K}\left(1.6 \mathrm{~cm}^{-1} / \mathrm{K}\right)$. Two excitation lines at $\lambda_{1}=487.6 \mathrm{~nm}$ and $\lambda_{2}=487.9 \mathrm{~nm}$ with a spectral distance of about $13 \mathrm{~cm}^{-1}$ using heatsink temperatures at $30{ }^{\circ} \mathrm{C}$ and $38{ }^{\circ} \mathrm{C}$ are 

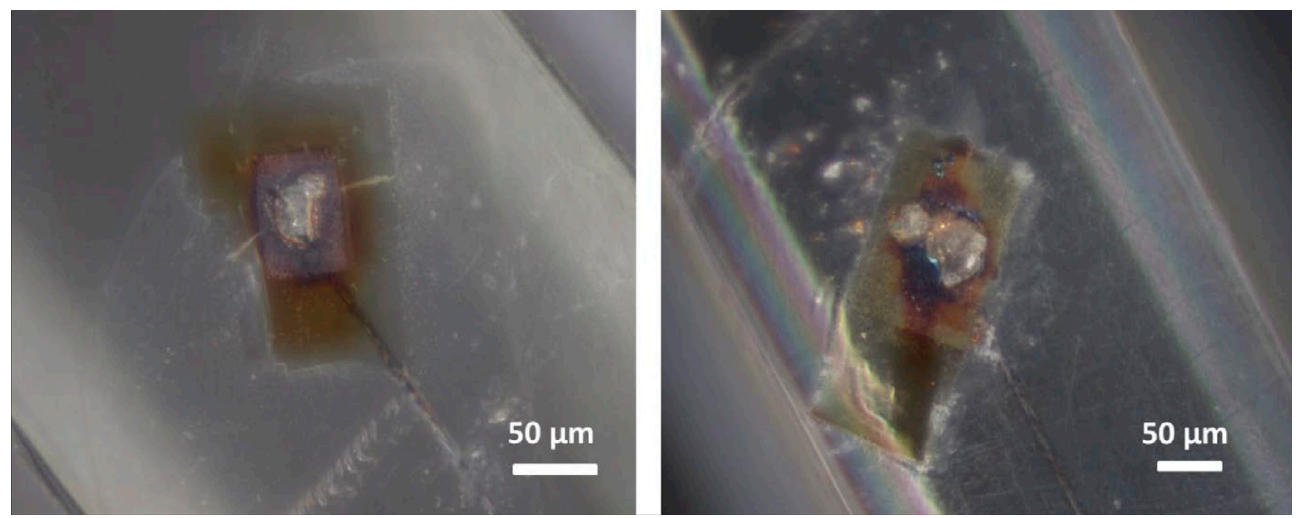

Fig. 1. Microscopic image of the Hayabusa particle \#39 (left) and \#68 (right).

Table 1

Investigation techniques applied to Hayabusa particles \#39 and \#68 and the corresponding sample handling before Raman spectroscopy was applied.

\begin{tabular}{|c|c|c|}
\hline Type of analysis & Hayabusa RA-QD-02-0039 & Hayabusa RA-QD-02-0068 \\
\hline $\begin{array}{l}\text { X-ray tomography ( } 7 \mathrm{keV} \text {, } \\
8 \mathrm{keV} \text { ) (Tsuchiyama et al., } \\
2011 \text { ) }\end{array}$ & $\begin{array}{l}\text { - exposed to Earth } \\
\text { atmosphere } \\
\text {-attached to carbon fiber } \\
\text { with resin }\end{array}$ & $\begin{array}{l}\text { • exposed to Earth } \\
\text { atmosphere } \\
\text { •attached to carbon fiber } \\
\text { with resin }\end{array}$ \\
\hline $\begin{array}{l}\text { SIMS analysis of O (and Mg } \\
\text { for \#68) isotopes } \\
\text { (Yurimoto et al., 2011) }\end{array}$ & $\begin{array}{l}\text { •exposed to Earth } \\
\text { atmosphere } \\
\text {-Au sputter coating } \\
\sim 60 \mathrm{~nm} \text { thickness for } \\
\text { charge compensation } \\
\text {-Sputtering by high energy } \\
\mathrm{Ga} \text { and O ion beams }\end{array}$ & $\begin{array}{l}\text {-exposed to Earth } \\
\text { atmosphere } \\
\text {-Au sputter coating } \\
\sim 60 \text { nm thickness for } \\
\text { charge compensation } \\
\text {-Sputtering by high energy } \\
\text { Ga ion beams }\end{array}$ \\
\hline XRD (Nakamura et al., 2011) & $\begin{array}{l}\text {-attached to carbon fiber } \\
\text { with resin }\end{array}$ & $\begin{array}{l}\text {-attached to carbon fiber } \\
\text { with resin }\end{array}$ \\
\hline $\begin{array}{l}\text { FE-SEM, FE-EPMA (Naka- } \\
\text { mura et al., 2011) }\end{array}$ & $\begin{array}{l}\text { •exposed to Earth } \\
\text { atmosphere } \\
\text { •polished (Sample sheet, } \\
\text { 2011) }\end{array}$ & $\begin{array}{l}\text { •exposed to Earth } \\
\text { atmosphere } \\
\text { •polished by M cross } \\
\text { (Sample sheet, 2011) }\end{array}$ \\
\hline & $\begin{array}{l}\bullet \text { C-coated (20 nm } \\
\text { thickness) }\end{array}$ & $\begin{array}{l}\text {-C-coated (20 nm } \\
\text { thickness) }\end{array}$ \\
\hline
\end{tabular}

selected for our SERDS experiments, respectively. The heatsink temperature is controlled and stabilized with a long term stability of $\pm 0.01 \mathrm{~K}$ corresponding to a wavelength stability of about $\pm 0.02 \mathrm{~cm}^{-1}$ for the excitation source providing reliability without the necessity of a spectral recalibration of the measurement system. Comparable excitation powers for both laser lines were achieved using neutral density (ND) filters in the collimated beam before coupling the laser light into the polarizationmaintained single-mode fiber. This enabled us to apply it as an excitation source for SERDS (Maiwald et al., 2009a). The collimated beam at $488 \mathrm{~nm}$ was coupled via an aspheric lens (focal length $f=8 \mathrm{~mm}$, numerical aperture $(\mathrm{NA})=0.5$ ) into a polarization-maintained single-mode fiber. This excitation fiber was connected to a confocal Raman microscope (WITec Alpha 300) with a thermo-electrically cooled $1024 \times 127$ pixel ANDOR iDUS DV401 CCD camera. A $20 \times$ objective $(N A=0.6)$ was used to focus the laser light on the sample and collect the backscattered photons. The acquisition time was adjusted between $2 \mathrm{~s}$ and $10 \mathrm{~s}$ per measured spectrum to avoid detector saturation with the sample under investigation. The detection fiber with core diameter of $50 \mu \mathrm{m}$ defined the entrance slit width of the spectrometer. The spectrometer was equipped with a 600 grooves/mm grating and allowed obtaining a Raman spectrum in the Stokes shifted spectral range up to $3900 \mathrm{~cm}^{-1}$. The spectral resolution was less than $16 \mathrm{~cm}^{-1}$. The power applied to the sample was kept between $0.2 \mathrm{~mW}$ and $0.4 \mathrm{~mW}$ in order to avoid sample heating (Böttger et al., 2017) and not to exceed the damage threshold of the sample. The laser beam diameter was about $1 \mu \mathrm{m}$.

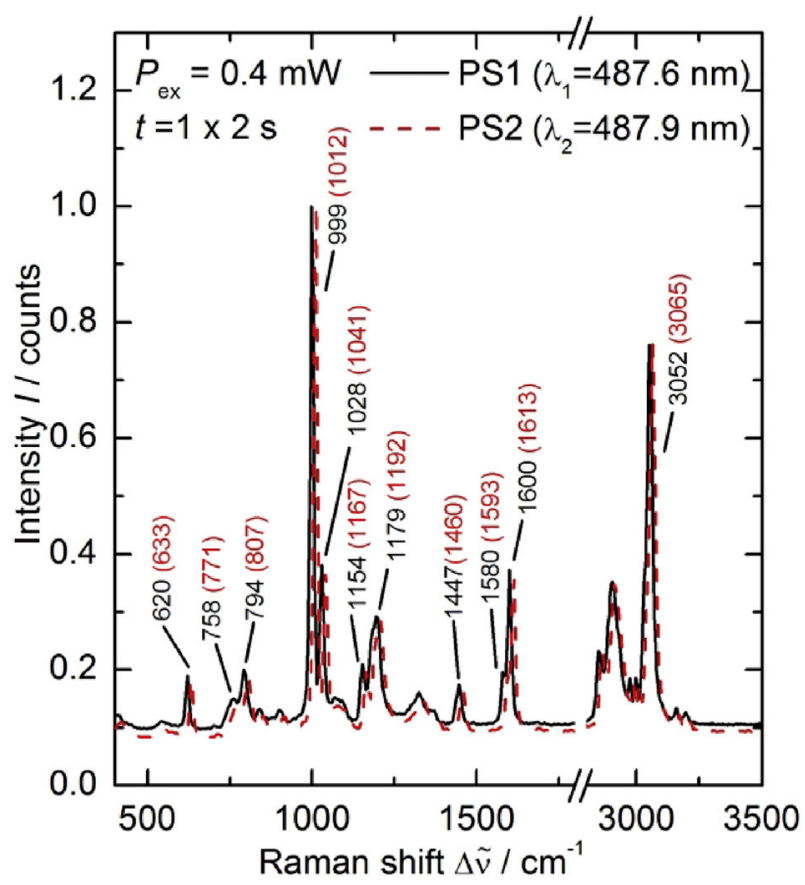

Fig. 2. Raman spectra of polystyrene (PS). PS1 and PS2 correspond to the Raman spectra excited with $\lambda_{1}$ and $\lambda_{2}$, respectively. The Raman shift numbers are given relative to laser excitation wavelength $\lambda_{1}$. The laser power on the sample was $0.4 \mathrm{~mW}$ and the acquisition time per spectrum was $2 \mathrm{~s}$.

\section{Results}

For spectral calibration of the Raman spectrometer, polystyrene (PS) was used. PS is well investigated by Raman spectroscopy (Schrader, 1989) and is used as Raman shift standards for spectrometer calibration (ASTM E 1840). In Fig. 2 Raman spectra of PS using both excitation lines at $\lambda_{1}=487.6 \mathrm{~nm}$ (black, solid, PS1) and $\lambda_{2}=487.9 \mathrm{~nm}$ (red, dashed, PS2) are presented. Here, the excitation power was $0.4 \mathrm{~mW}$. Each spectrum was obtained with $2 \mathrm{~s}$ integration time. The intensity of each spectrum was normalized to 1 at $999 \mathrm{~cm}^{-1}$ for PS1 and $1012 \mathrm{~cm}^{-1}$ for PS2. The band positions of PS1 are correlated to literature values (Schrader, 1989) and are used for spectral calibration. The separation of the laser wavelengths corresponds to $13 \mathrm{~cm}^{-1}$.

After this calibration procedure the Hayabusa samples were investigated. Raman spectra were measured at different positions on both Hayabusa particles (Fig. 3). For SERDS at each point two spectra were taken directly one after the other with the two excitation wavelengths $\lambda_{1}$ and $\lambda_{2}$. Typical conventional Raman spectra are shown in Fig. 4.

These classical Raman spectra are dominated by strong fluorescence 

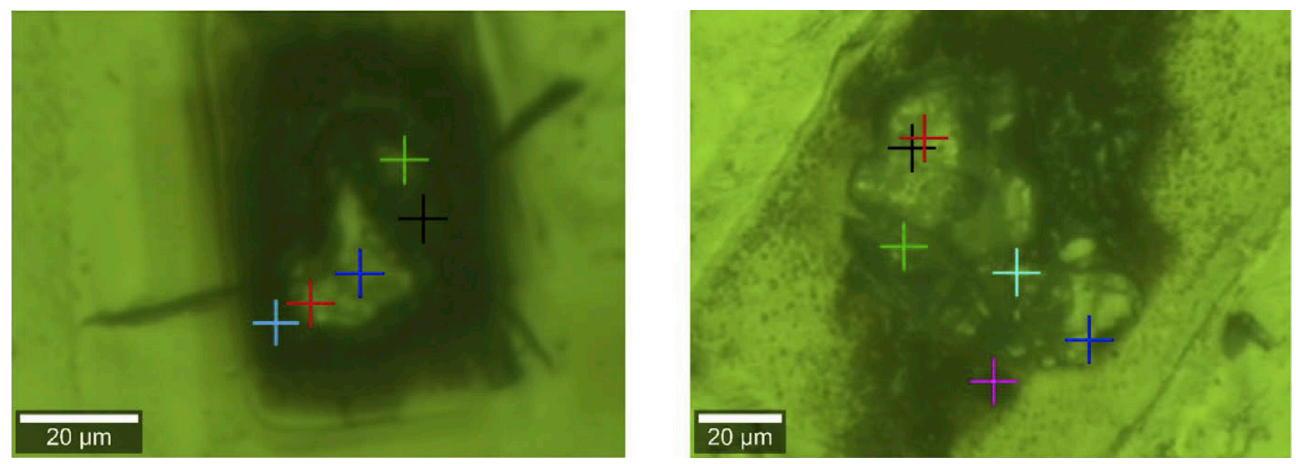

Fig. 3. Raman measurement positions (indicated by crosses) on the Hayabusa particles \#39 (left) and \#68 (right).
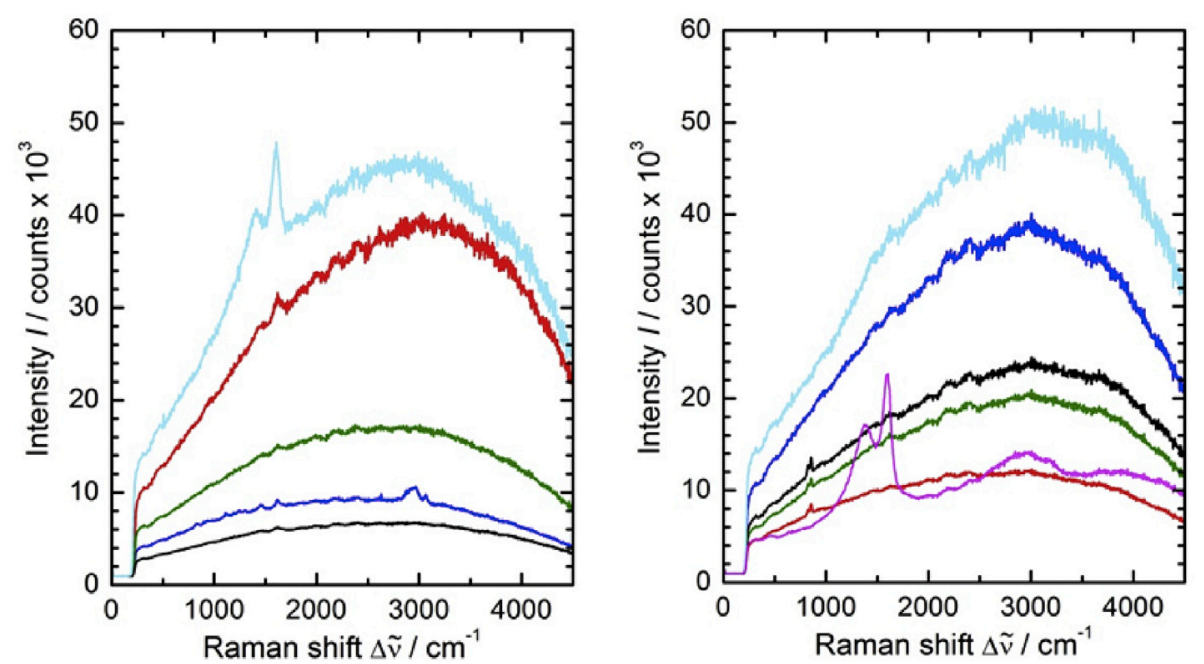

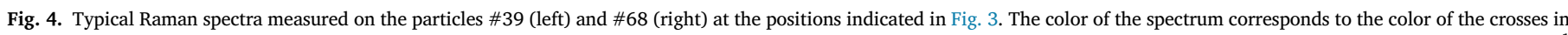

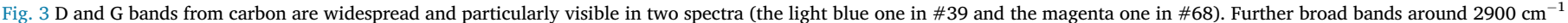
probably due to the presence of organic compounds are visible in two spectra (the blue one in \#39 and the magenta one in \#68).

and only few predominant features are seen. These features are registered in the Raman spectra taken at the edges of the particles (light blue spectrum for particle \#39 and magenta spectrum for particle \#68). They correspond to carbon with the prominent positions of the $\mathrm{D}$ and $\mathrm{G}$ bands around $1376 \mathrm{~cm}^{-1}$ and $1593 \mathrm{~cm}^{-1}$ (Escribano et al., 2001; Bokobza et al., 2015). These spectra can be assigned to remnants of the carbon coating, which was applied during sample preparation of the particles (see Table 1) and remained, despite polishing, in small gaps and cracks. Furthermore, for particle \#68 three spectra (green, black and red in Fig. 4) show very small features around $822 \mathrm{~cm}^{-1}$ and $852 \mathrm{~cm}^{-1}$ which originate from olivine. In addition, small features between $1600 \mathrm{~cm}^{-1}$ and $3000 \mathrm{~cm}^{-1}$ can be seen in all spectra.

The SERDS spectrum in Fig. 5 was obtained from the center region of Hayabusa sample \#68. It is seen that in the raw spectra (green and black curves) the signals from olivine at $822 \mathrm{~cm}^{-1}$ and $852 \mathrm{~cm}^{-1}$ are shifted by $13 \mathrm{~cm}^{-1}$ with respect to each other. This is the difference of the laser wavelengths. In contrast to the conventional Raman spectra the SERDS spectra show the doublet feature and further bands of olivine which are clearly resolved and not masked by fluorescence anymore (red curve). The Mg content of the olivine can be determined from the position of the two peaks (Kuebler et al., 2006; Böttger et al., 2014a,b). Within the measurement uncertainty it is equal to those values obtained for other Hayabusa particles (Böttger et al., 2014a,b). In contrast to olivine the features between $1600 \mathrm{~cm}^{-1}$ and $3000 \mathrm{~cm}^{-1}$ are not shifted in the spectra obtained with the two different wavelengths. Therefore, they can be classified as artifacts. They may arise from CCD structured noise or the cover glass of the sample container, which is not perfectly plane parallel and orthogonal to the optical axis of the microscope. It is worth noting that the SERDS method efficiently separates Raman signals from background signals such as fluorescence, ambient light, fixed-pattern noise, and artifacts as can be seen from the SERDS spectrum in Fig. 5.

Some Raman spectra (Fig. 6, black curve) and the reconstructed SERDS spectra (red curve) of particle \#39 show small features at about $1450 \mathrm{~cm}^{-1}$ and $1600 \mathrm{~cm}^{-1}$. These cannot be assigned to carbon, although this region of the Raman shifts corresponds to CC stretching vibrations. The reason is that additional features are visible in the $\mathrm{CH}$ - vibration region above $2800 \mathrm{~cm}^{-1}$. These occur if organic compounds are involved. From the particle treatment history it is known that the particles were transported in membrane boxes. Therefore, we measured the Raman spectrum of the membrane foil for comparison (blue curve). Some dominant Raman bands of the spectrum measured on the particle are comparable with the Raman lines of the membrane foil.

\section{Summary and conclusions}

Raman and SERDS measurements were performed in order to investigate extraterrestrial particles from the asteroid Itokawa. For the experiments a compact dual-wavelength diode laser based light source at $488 \mathrm{~nm}$ was used as the excitation source and was coupled via a singlemode fiber to a confocal Raman microscope. With this setup two particles were analyzed. Prior to the Raman measurements both particles experienced several treatments that influenced their Raman spectra and 


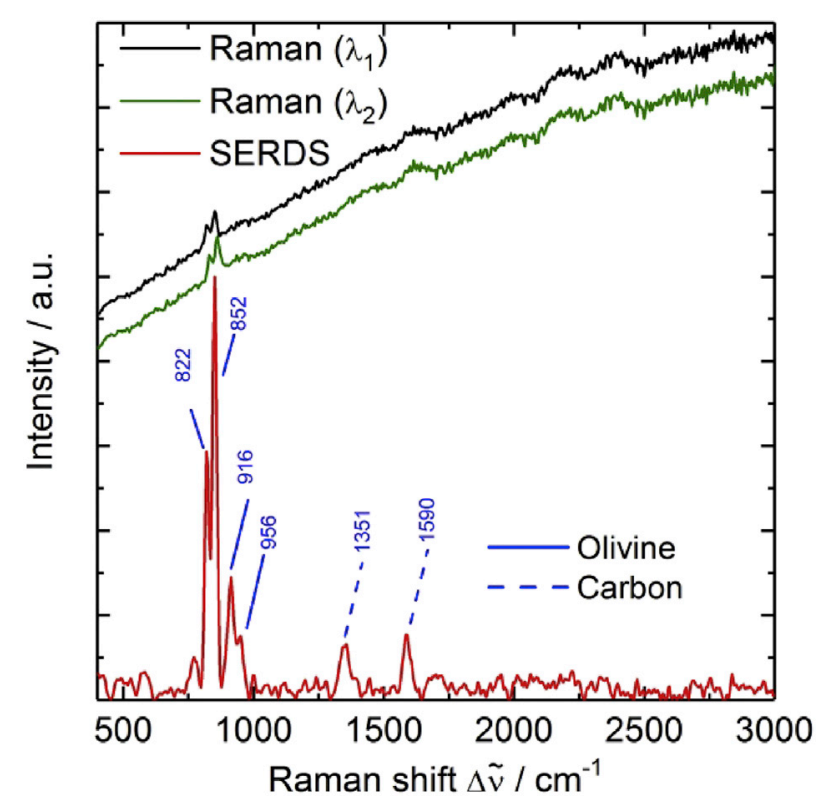

Fig. 5. Average of ten Raman spectra of the particle \#68 for the two laser excitation wavelengths $\lambda_{1}$ and $\lambda_{2}$ (black and green, respectively. The intensity offset is for clarity.) and reconstructed SERDS spectrum for the black cross position on the sample. Excitation power at the sample was $0.2 \mathrm{~mW}$ and $10 \mathrm{~s}$ integration time for each Raman spectrum.

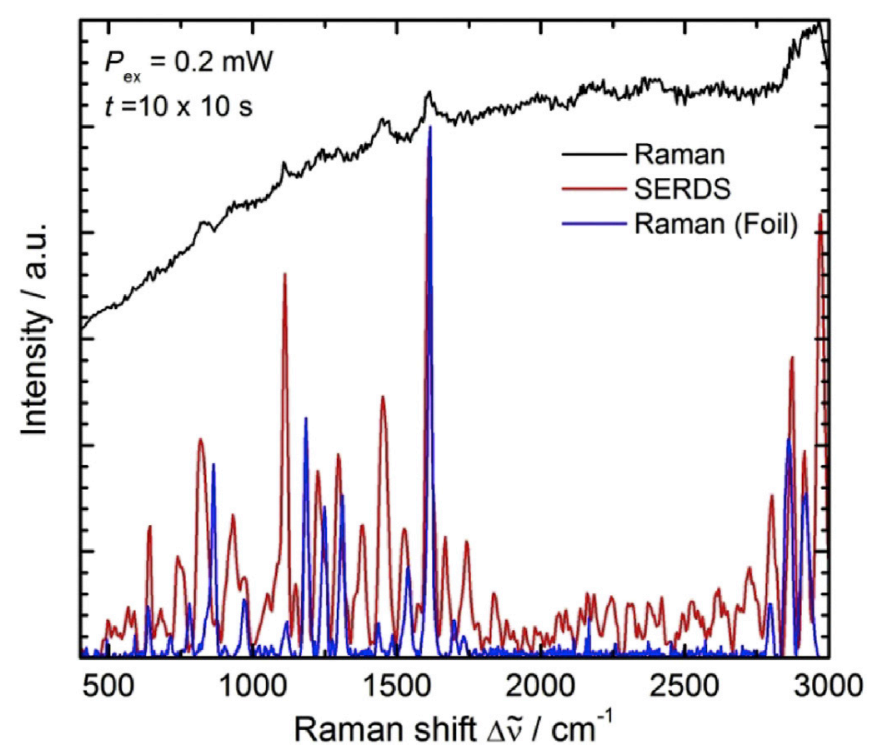

Fig. 6. Average of ten Raman spectra from Hayabusa particle \#39 (black), and deduced SERDS spectrum (red) for the dark blue cross position on the sample (Fig. 3). The sample was excited with $0.2 \mathrm{~mW}$ and the measurement was performed with $10 \mathrm{~s}$ integration time for each Raman spectrum. The Raman spectrum from membrane foil (blue) is shown for comparison.

particularly the fluorescence response rather strongly. This was ideal to demonstrate the advantages of SERDS. Using SERDS several improvements with respect to conventional Raman spectroscopy have been realized. First of all the Raman features of the minerals, in this case olivine, were free of baseline shifts and their frequency position could be identified more reliably. Secondly, artifacts could be distinguished from Raman signals, since the artifacts did not depend on the excitation wavelength and therefore disappear in the SERDS spectrum. Finally, the assignment of the Raman signal of carbonaceous material is more certain.
Hence, this feasibility study showed that it is possible to reduce the impact of fluorescence that dominates the conventional Raman spectra and to distinguish between the original mineralogy of the particles and the remnants from the various treatments the particles experienced during their investigation history.

SERDS efficiently separates Raman signals from background signals such as fluorescence, ambient light and artifacts (Yan et al., 2016a,b; Maiwald et al., 2016), e.g., fixed pattern detector noise, and can extract weak Raman signals for identification of substances. For in-situ space exploration this method is of high relevance, because of its spectroscopic advantages as well as of its relatively simple implementation. Furthermore, SERDS is comparatively easy to adapt to existing continuous wave systems and will be a worthwhile extension for continuous wave Raman spectroscopy for both planetary exploration and laboratory-based measurements.

\section{Acknowledgment}

The authors thank M. Greiner-Bär for technical support and JAXA for the allocation of samples from asteroid Itokawa. F. H. acknowledges support from the Helmholtz Research School on Security Technologies.

\section{References}

Bokobza, L., Bruneel, J.-L., Couzi, M., 2015. Raman spectra of carbon-based materials (from graphite to carbon black) and of some silicone composites. C 1, 77-94.

Böttger, U., Alwmark, C., Bajt, S., Busemann, H., Gilmour, J.D., Heitmann, U., Hübers, H.W., Meier, M.M.M., Pavlov, S.G., Schade, U., Spring, N.H., Weber, I., 2014a. Raman spectroscopy of extraterrestrial particles from asteroid Itokawa. In: Proceedings XXIV. ICORS 2014, Jena.

Böttger, U., Alwmark, C., Bajt, S., Busemann, H., Gilmour, J.D., Heitmann, U., Hübers, H.W., Meier, M.M.M., Pavlov, S.G., Schade, U., Spring, N.H., Weber, I., 2014b. Mineralogy and structure of Hayabusa particles using Raman micro-spectroscopy. In: EPSC 2014. Abstract \#312.

Böttger, U., Pavlov, S.G., Deßmann, N., Hanke, F., Weber, I., Fritz, J., Hübers, H.-W., 2017. Laser-induced alteration of Raman spectra for micron-sized solid particles. Planet. Space Sci. 138, 25-32.

Busemann, H., Alwmark, C., Bajt, S., Böttger, U., Gilmour, J.D., Heitmann, U., Hübers, H.W., Meier, M.M.M., Pavlov, S., Schade, U., Spring, N.H., Weber, I., 2013. A consortium status report: the characterisation of the asteroid Itokawa regolith - a correlated study by X-ray tomography, micro-Raman spectroscopy, and highsensitivity noble gas analysis. In: Lunar \& Planetary Science Conference XLIV, 2013. Abstract \#2243.

Busemann, H., Meier, M.M.M., Altmann, F., Alwmark, C., Bajt, S., Beyersdorfer, J., Böttger, U., Crowther, S., Gilmour, J., Heitmann, U., Hübers, H.-W., Maden, C., Marone, F., Pavlov, S., Schade, U., Spring, N., Stampanoni, M., Weber, I., 2015. New noble gas data and further examinations of dust from asteroid ITOKAWA. In: 46 Lunar \& Planetary Science Conference 2015. Abstract \#2113.

Ebihara, M., Sekimoto, S., Shirai, N., Hamajima, Y., Yamamoto, M., Kumagai, K., Oura, Y., Ireland, T.R., Kitajima, F., Nagao, K., Nakamura, T., Naraoka, H., Noguchi, T., Okazaki, R., Tsuchiyama, A., Uesugi, M., Yurimoto, H., Zolensky, M.E., Abe, M., Fujimura, A., Mukai, T., Yada, T., 2011. Neutron activation analysis of a particle returned from asteroid Itokawa. Science 333, 1119. http://dx.doi.org/10.1126/ science. 1207865 .

Escribano, R., Sloan, J.J., Siddique, N., Sze, N., Dudev, T., 2001. Raman spectroscopy of carbon-containing particles. Vib. Spectrosc. 26, 179-186.

Hooijschuur, J.-H., Iping Petterson, I.E., Davies, G.R., Gooijer, C., Ariese, F., 2013. Time resolved Raman spectroscopy for depth analysis of multi-layered mineral samples. J. Raman Spectrosc. 44 (11), 1540-1547. http://dx.doi.org/10.1002/jrs.4369.

Kuebler, K., Jolliff, B.L., Wang, A., Haskin, L.A., 2006. Extracting olivine (Fo-Fa) composition from Raman spectral peak positions. Geochim. Cosmochim. Acta 70, 6201-6222.

Maiwald, M., Schmidt, H., Sumpf, B., Güther, R., Erbert, G., Kronfeldt, H.-D., Tränkle, G., 2009a. Microsystem light source at $488 \mathrm{~nm}$ for shifted excitation resonance raman difference spectroscopy. Appl. Spec. 63 (No. 11), 1283-1287.

Maiwald, M., Jedrzejczyk, D., Sahm, A., Paschke, K., Güther, R., Sumpf, B., Erbert, G., Tränkle, G., 2009b. Second-harmonic-generation microsystem light source at $488 \mathrm{~nm}$ for Raman spectroscopy. Opt. Lett. 34 (No. 2), 217-219.

Maiwald, M., Eppich, B., Fricke, J., Ginolas, A., Bugge, F., Sumpf, B., Erbert, G., Tränkle, G., 2014. Dual-wavelength Y-Branch distributed Bragg reflector diode laser at 785 Nanometers for shifted excitation Raman difference spectroscopy. Appl. Spectrosc. 68, 838 .

Maiwald, M., Müller, A., Sumpf, B., Erbert, G., Tränkle, G., 2015. Capability of shifted excitation Raman difference spectroscopy under ambient daylight. Appl. Opt. 54 (No. 17), 5520-5524.

Maiwald, M., Müller, A., Sumpf, B., Tränkle, G., 2016. A portable shifted excitation Raman difference spectroscopy system: device and field demonstration. J. Raman Spectrosc. 47 (No. 10), 1180-1184. 
Matousek, P., Towrie, M., Stanley, A., Parker, A.W., 1999. Efficient rejection of fluorescence from Raman spectra using picosecond Kerr gating. Appl. Spectrosc. 53, 1485-1489.

May, L., Mattingly, R., 2011. Mars sample return as a campaign. In: IEEE Aerospace Conference, pp. 1-13. http://dx.doi.org/10.1109/AERO.2011.5747287.

McCain, S.T., Willett, R.M., Brady, D.J., 2008. Multi-excitation Raman spectroscopy technique for fluorescence rejection. Opt. Exp. 16, 10975.

Meier, M.M.M., Alwmark, C., Bajt, S., Böttger, U., Busemann, H., Fujiya, W., Gilmour, J., Heitmann, U., Hoppe, P., Hübers, H.-W., Marone, F., Ott, U., Pavlov, S., Schade, U. Spring, N., Stampanoni, M., Weber, I., 2014. A precise cosmic ray exposure age for an olivine grain from the surface of near-earth asteroid (25143) ITOKAWA. In: Lunar \& Planetary Science Conference XLV, 2014. Abstract \#1247.

Mosier-Boss, P.A., Liebermann, S.H., Newberry, R., 1995. Fluorescence rejection in Raman spectroscopy by shifted-spectra, edge detection, and FFT filtering techniques. Appl. Spectrosc. 49, 630 .

Nakamura, T., Noguchi, T., Tanaka, M., Zolensky, M.E., Kimura, M., Tsuchiyama, A., Nakato, A., Ogami, T., Ishida, H., Uesugi, M., Yada, T., Shirai, K., Fujimura, A., Okazaki, R., Sandford, S.A., Ishibashi, Y., Abe, M., Okada, T., Ueno, M., Mukai, T., Yoshikawa, M., Kawaguchi, J., 2011. Itokawa dust particles: a direct link between SType asteroids and ordinary chondrites. Science 333, 1113. http://dx.doi.org/ 10.1126/science.1207758.

Naraoka, H., Mita, H., Hamase, K., Mita, M., Yabuta, H., Saito, K., Fukushima, K., Kitajima, F., Sandford, S.A., Nakamura, T., Noguchi, T., Okazaki, R., Agao, K., Ebihara, M., Yurimoto, H., Tsuchiyama, A., Abe, M., Shirai, K., Ueno, M., Yada, T., Ishibashi, Y., Okada, T., Fujimura, A., Mukai, T., Yoshikawa, M., Kawaguchi, J., 2012. Preliminary organic compound analysis of microparticles returned from Asteroid 25143 Itokawa by the Hayabusa mission. Geochem. J. 46, 61-72.

Rull, F., Maurice, S., Diaz, E., Lopez, G., Catala, A., RLS Team, 2013. Raman laser spectrometer (RLS) for ExoMars 2018 rover mission: current status and science operation mode on powdered samples. In: 44th Lunar and Planetary Science Conference, Held March 18-22, 2013. Woodlands, Texas, p. 3110. Abstract No. 1719.

Sample sheet of particle RA-QD02-0039 provided by JAXA (2011).

Schrader, B., 1989. In: Schrader, B. (Ed.), "N-03", in Raman-, Infrared Atlas of Organic Compounds. Wiley-CVH, 1989. N-03.

Shreve, P., Cherepy, N.J., Mathies, R.A., 1992. Effective rejection of fluorescence interference in raman spectroscopy using a shifted excitation difference technique. Appl. Spectrosc. 46, 707.

Tachibana, S., Abe, M., Arakawa, M., Fujimoto, M., Iijima, Y., Ishiguro, M., Kitazato, K., Kobayashi, N., Namiki, N., Okada, T., Okazaki, R., Sawada, H., Sugita, S., Takano, Y.,
Tanaka, S., Watanabe, S., Yoshikawa, M., Kuninaka, H., the Hayabusa2 Project Team, 2014. Hayabusa2: scientific importance of samples returned from C-type near-earth asteroid (162173) 1999 JU3. Geochem. J. 48 (No. 6), 571-587.

Tsuchiyama, A., Uesugi, M., Matsushima, T., Michikami, T., Kadono, T., Nakamura, T., Uesugi, K., Nakano, T., Sandford, S.A., Noguchi, R., Matsumoto, T., Matsuno, J., Nagano, T., Imai, Y., Takeuchi, A., Suzuki, Y., Ogami, T., Katagiri, J., Ebihara, M., Ireland, T.R., Kitajima, F., Nagao, K., Naraoka, H., Noguchi, T., Okazaki, R., Yurimoto, H., Zolensky, M.E., Mukai, T., Abe, M., Yada, T., Fujimura, A., Yoshikawa, M., Kawaguchi, J., 2011. Three-dimensional structure of Hayabusa samples: origin and evolution of Itokawa regolith. Science 333, 1125. http:// dx.doi.org/10.1126/science.1207807.

Tuschel, D., 2016. Selecting an excitation wavelength for Raman spectroscopy. Spectroscopy 31 (3), 14-23.

Wei, D., Chen, S., Liu, Q., 2015. Review of fluorescence suppression techniques in Raman spectroscopy. Appl. Spectrosc. Rev. 50, p 387.

Witze, A., 2014. NASA plans mars sample-return rover. Nature 509 (7500), 272.

Yan, Y., Wang, A., Wei, J., 2016a. Shifted excitation Raman differentiated spectroscopy for planetary surface exploration. In: XII Georaman 2016. Novosibirsk, Russia.

Yan, C., Wang, A., Wei, J., 2016b. Shifted excitation Raman differentiated spectroscopy (SERDS) for planetary surface exploration. In: 47 LPSC 2016. Abstract \#2210.

Yoshikawa, M., Kuninaka, H., Inaba, N., Watanabe, S., Tsuda, Y., Tanaka, S., Fujimoto, M., Abe, M., Sugita, S., Namiki, N., Kitazato, K., Arakawa, M., Tachibana, S., Ishiguro, M., Ikeda, H., Fujiwara, A., Kawaguchi, J., 2015. The science of sample return missions Hayabusa and Hayabusa2. In: 11th Low Cost Planetary Missions Conference, June 9-11, Berlin, Germany.

Yurimoto, H., Abe, K., Abe, M., Ebihara, M., Fujimura, A., Hashiguchi, M., Hashizume, K., Ireland, T.R., Itoh, S., Katayama, J., Kato, C., Kawaguchi, J., Kawasaki, N., Kitajima, F., Kobayashi, S., Meike, T., Mukai, T., Nagao, K., Nakamura, T., Naraoka, H., Noguchi, T., Okazaki, R., Park, C., Sakamoto, N., Seto, Y., Takei, M., Tsuchiyama, A., Uesugi, M., Wakaki, S., Yada, T., Yamamoto, K., Yoshikawa, M., Zolensky, M.E., 2011. Oxygen isotopic compositions of asteroidal materials returned from Itokawa by the Hayabusa mission. Science 333, 1116. http://dx.doi.org/ 10.1126/science.1207776.

Zelenyi, L., Mitrofanov, I., Petrukovich, A., Khartov, V., Martynov, M., Lukianchikov, A., 2014. Russian plans for lunar investigations. Stage 1. In: EPSC 2014. Abstract \#702.

Zhao, J., Carrabba, M.M., Allen, F., 2002. Automated fluorescence rejection using shifted excitation Raman difference spectroscopy. Appl. Spectrosc. 56, 834.

Zheng, Y., Ouyang, Z., Li, C., Liu, J., Zou, Y., 2008. China's lunar exploration program: present and future. Planet. Space Sci. 56 (7), 881-886. 